\title{
SANT'AGOSTINO E LE SCIENZE
}

\author{
CLAUDIO CITRINI (*) \\ Nota presentata dal m.e. Carlo Domenico Pagani \\ (Adunanza del 6 ottobre 2016)
}

SunTO. - Si riportano numerosi esempi delle conoscenze scientifiche di Sant'Agostino, che dimostrano la sua costante ricerca e adesione alle verità della ragione, in un rapporto complesso ma mai conflittuale con quelle della fede. Con ragionamenti a volte nitidi, altre volte contorti o erronei egli cerca di superare ogni contrasto con l'interpretazione, letterale o allegorica, delle Scritture.

$* * *$

ABSTRACT. - Several examples of Saint Augustin's learning in sciences are reported. They show his constant research of the truth both in human rational knowledge and in faith, which must never be in disagreement. He always tries to overcome, with sometimes straightforward and sometimes sophistic arguments, any inconsistency of the scientific knowledge with the literal or allegoric interpretation of the Holy Scriptures.

\section{Fede E RAgione}

Non si può comprendere il rapporto di Sant'Agostino con le scienze se non si tiene in conto il suo percorso culturale e spirituale. La sua formazione comprendeva tutte le arti che poi furono dette liberali, e, per quanto portato per la retorica, in cui acquisì un tale prestigio da meritarsi la nomina a maestro di retorica nella città allora capitale imperiale, non dimenticò mai la sua preparazione scientifica. Quando, venuto a Milano per assumere la carica, incontrò il vescovo Ambrogio, la sua vita ebbe una svolta, culminata con la conversione e il battesimo, tutta

(*) Istituto Lombardo Accademia di Scienze e Lettere, Milano, Italia.

E-mail: claudio.citrini@polimi.it 
la sua vita. La sua produzione apologetica, tuttavia, non fu mai in contrasto con la verità scientifica appresa, ma si sforzò sempre di conciliarla con la verità di fede.

Nella sua catechesi del mercoledì 21 novembre 2012 papa Benedetto XVI descrisse magistralmente il ruolo di Agostino nel percorso di razionalizzazione della fede: "Oggi in questa catechesi vorrei soffermarmi sulla ragionevolezza della fede in Dio. La tradizione cattolica sin dall'inizio ha rigettato il cosiddetto fideismo, che è la volontà di credere contro la ragione. Credo quia absurdum (credo perché è assurdo) non è formula che interpreti la fede cattolica. Dio, infatti, non è assurdo, semmai è mistero. Il mistero, a sua volta, non è irrazionale, ma sovrabbondanza di senso, di significato, di verità....

Sant'Agostino, prima della sua conversione, cerca con tanta inquietudine la verità, attraverso tutte le filosofie disponibili, trovandole tutte insoddisfacenti. La sua faticosa ricerca razionale è per lui una significativa pedagogia per l'incontro con la Verità di Cristo. Quando dice: «comprendi per credere e credi per comprendere» (Discorso 43, 9), è come se raccontasse la propria esperienza di vita. Intelletto e fede, dinanzi alla divina Rivelazione non sono estranei o antagonisti, ma sono ambedue condizioni per comprenderne il senso, per recepirne il messaggio autentico, accostandosi alla soglia del mistero. Sant'Agostino, insieme a tanti altri autori cristiani, è testimone di una fede che si esercita con la ragione, che pensa e invita a pensare. Su questa scia, Sant'Anselmo dirà nel suo Proslogion che la fede cattolica è fides quaerens intellectum, dove il cercare l'intelligenza è atto interiore al credere. Sarà soprattutto San Tommaso d'Aquino - forte di questa tradizione a confrontarsi con la ragione dei filosofi, ...”.

Vediamo dunque alcuni esempi.

\subsection{Razionalità del creato}

Il mondo, per Agostino, è fatto con tale perfezione che non può assolutamente essere dovuta al caso. Nel De Ordine (I, 1.2) egli scrive: "O le membra di animali anche piccolissimi sono strutturate dal caso in dimensioni tanto proporzionate ed esatte; ovvero si ammette che deriva da un principio razionale che non può esser prodotto dal caso; o infine noi oseremmo, per pregiudizi di vana filosofia, non attribuire all'occulta legge del divino potere l'ordine che ammiriamo in ogni essere nella successione di tutti i fenomeni naturali e indipendentemente dalla 
razionale produttività dell'uomo. ... Ma a questo proposito supponiamo che un tale abbia la vista tanto limitata che in un pavimento a mosaico il suo sguardo possa percepire soltanto le dimensioni di un quadratino per volta. Egli rimprovererebbe all'artista l'imperizia nell'opera d'ordinamento e composizione nella convinzione che le diverse pietruzze sono state maldisposte. Invece è proprio lui che non può cogliere e rappresentarsi in una visione d'insieme i pezzettini armonizzati in una riproduzione d'unitaria bellezza. ... La medesima condizione si verifica per le persone incolte. Incapaci di comprendere e riflettere sull'universale e armonico ordinamento delle cose, se qualche aspetto, che per la loro immaginazione è grande, li urta, pensano che nell'universo esiste una grande irrazionalità".

Dunque i dettagli, visti separatamente o non compresi, non permettono di ammirare il meraviglioso quadro della natura, che solo la legge divina ha potuto concepire e attuare.

\subsection{Razionalità dell'uomo}

Tutto dunque è razionale nel creato, e anche gli animali agiscono secondo un istinto dettato dalla ragione. Naturalmente, l'uomo è a un livello più elevato in quanto dotato di consapevolezza.

Ancora nel De Ordine (II, 19. 49) leggiamo: "Da molti elementi sparsi disordinatamente e poi radunati secondo una struttura unitaria io costruisco una casa. Io valgo di più perché la faccio ed essa è fatta. ... Ma non per lo stesso motivo valgo di più della rondine e dell'ape poiché la prima tanto ingegnosamente costruisce i nidi e la seconda i favi. Valgo di più perché sono un animale dotato di ragione. Ma se la ragione consiste nelle misure razionalmente disposte, forse che la costruzione degli uccelli non è misurata proporzionatamente e convenientemente? Anzi ha esattezza matematica. Quindi valgo di più non perché eseguo opere matematicamente esatte ma perché conosco l'esattezza matematica. Ma allora? Gli uccelli possono realizzare cose matematicamente esatte pur non avendone scienza? Lo possono certamente. Da dove l'hanno appreso? Da quello stesso principio per cui anche noi adattiamo la lingua ai denti e al palato perché ne escano parole. Non pensiamo tuttavia nel parlare con quale movimento della bocca lo facciamo".

L'istinto, dunque, o comunque il comportamento appreso per mimesi e applicato senza controllo specifico, per Agostino è ragione inconsapevole. 


\subsection{Scienza e scienziati}

Purtroppo l'uomo talvolta si inorgoglisce della potenza del suo pensiero, e dimentica che essa non è altro che il riflesso della sapienza divina. Ecco un bel passo dalle Confessioni (Conf. V, 3. 4): "Investigando questi misteri con l'intelligenza e l'ingegno da te ricevuti, [gli astronomi] fecero molte scoperte, predissero con anticipo di molti anni le eclissi della luce del sole e della luna, con il giorno, l'ora e la misura in cui sarebbero avvenuti, senza errare nei calcoli. I fenomeni si verificarono puntualmente secondo le loro predizioni, ed essi misero per iscritto le leggi scoperte, tuttora consultate e usate per predire l'anno, il mese dell'anno, il giorno del mese, l'ora del giorno e la misura in cui la luce del sole e della luna scomparirà; e il fenomeno avverrà puntualmente secondo le predizioni. Il popolo ne è ammirato, gli ignari stupiti, gli esperti imbaldanziti ed esaltati. Ma se, lontani ed eclissati dalla tua luce per la loro empia superbia, prevedono con tanto anticipo l'offuscamento futuro del sole, non vedono però il loro, presente, poiché non ricercano con spirito religioso l'origine del proprio ingegno, con cui eseguono queste ricerche; o, se si scoprono tue creature, non si donano a te con slancio affinché tu conservi le tue creature".

Qui entra in gioco l'atteggiamento di Agostino. La scienza lo affascina, ma nulla conta senza la fede. È un mezzo per giungere a intuire che la razionalità del mondo è manifestazione di Dio, ma nulla conta nella storia della salvezza dell'uomo. Più avanti, a proposito degli errori scientifici dei Manichei, afferma (Conf. V, 5. 9): "Ascoltando qua o là un mio fratello cristiano, che in materia è inesperto e ha idee sbagliate, io considero le sue opinioni pazientemente né vedo come gli nuoccia l'ignorare accidentalmente la posizione e la condotta di enti corporei creati da te, allorché su di te, Signore, creatore di tutto, non ha opinioni sconvenienti. Gli nuocerebbe invece il pensare che questa scienza faccia parte proprio dell'insegnamento religioso e l'affermare con sfrontata ostinazione quanto ignora”.

Comunque la scienza ha valore di verità assoluta: "La scienza infatti non è costituita da una qualsiasi rappresentazione, ma da una rappresentazione tale che chi l'ha formulata non commetta errore e, anche se sottoposto a qualsiasi critica, non possa dubitare. Ne consegue la indiscussa verità dell'affermazione di alcuni filosofi che in nessuno essa si può ritrovare se non nel filosofante. Egli non deve soltanto aver 
conoscenza della dottrina che sostiene, ma trovarle anche un fondamento incrollabile" (Contra Academicos, I, 7. 19).

\subsection{Scienza e scrittura}

La scienza dunque ha le sue verità, ma anche la Scrittura è fonte di verità, e di livello superiore. Non ci può e non ci deve essere un contrasto tra le due. Ma se la scrittura è presa alla lettera, come facevano i Manichei, può anche affermare cose che alla scienza appaiono erronee. Questo era uno dei motivi per cui il giovane Agostino non si era accostato alla Scrittura come avrebbe voluto la madre Monica, profondamente cristiana.

Giunto a Milano, Agostino aveva appreso da Sant'Ambrogio a fare una lettura metaforica della Scrittura, per cavarne le verità più profonde che si celano in essa; tuttavia egli cercò sempre di salvare anche il significato letterale, pur affermando esplicitamente che esso non ha e non vuole avere un contenuto scientifico.

Per esempio, a proposito della forma del cielo vi sono passi delle Scritture in apparente contrasto: Nei salmi si legge "Qui extendit coelum sicut pellem" (Ps 103, 2), ma Isaia dice che "il cielo sta sospeso come una volta" (Is 40, 22 sec. LXX). La prima concezione, così ingenua, è ripresa in un affresco del 1246 che si trova nell'oratorio di San Silvestro adiacente alla chiesa romana dei Santi Quattro Coronati. Accanto alle storie di Costantino e di papa Silvestro I si può ammirare un Giudizio universale, nel quale un angelo arrotola il cielo stellato proprio come fosse un tappeto!

Il primo commento di Agostino è che il problema non è rilevante per la felicità e salvezza eterna: "Che importa infatti se il cielo racchiude da ogni parte, come una sfera, la terra mantenuta in equilibrio al centro del mondo oppure la copra come un disco solo dalla parte superiore? Ma qui è in gioco la credibilità della Scrittura per il motivo più volte da me ricordato" (De Gen. II, 9, 20).

Pertanto Agostino così argomenta: "Ecco perché è necessario dire in breve che i nostri agiografi conoscevano quanto è conforme alla verità per ciò che riguarda la figura del cielo, ma lo Spirito di Dio, che parlava per mezzo di essi, non ha voluto insegnare agli uomini queste cognizioni per nulla utili alla salvezza dell'anima".

Questo argomento, come è ben noto, comprese le due precedenti citazioni, sarà ripreso da Galileo, nella celebre Lettera a Cristina di 
Lorena: "E se l'istesso Spirito Santo a bello studio ha pretermesso d'insegnarci simili proposizioni, come nulla attenenti alla sua intenzione, ciò è alla nostra salute, come si potrà adesso affermare, che il tener di esse questa parte, e non quella, sia tanto necessario che l'una sia de Fide, e l'altra erronea? ... Io qui direi che quello che intesi da persona ecclesiastica costituita in eminentissimo grado [il cardinal Baronio], ciò è l'intenzione delle Spirito Santo essere d'insegnarci come si vadia al cielo, e non come vadia il cielo".

Tuttavia, quando la scienza si pronuncia con certezza, bisogna rivedere l'interpretazione della Scrittura, in modo da evitare ogni conflitto: "Ma se per caso quelli [che attribuiscono al cielo la figura d'una sfera] potessero provare la loro opinione con argomenti di cui non si dovrebbe dubitare, bisognerebbe dimostrare che l'immagine della pelle usata dai nostri Libri sacri non è in contraddizione con quelle argomentazioni razionali" (De Gen. II, 9. 21). O anche: "Qualora ciò si avverasse, non era quello il senso della sacra Scrittura, ma un'opinione dell'umana ignoranza" (De Gen. I, 19, 38).

Non si può non rilevare la consonanza con il pensiero del cardinale San Roberto Bellarmino, a proposito di un tema analogo, quello del sistema eliocentrico, ovviamente contrario alle teorie geocentriche adottate nella Scrittura: "Dico che quando ci fusse vera dimostrazione che il sole stia nel centro del mondo e la terra nel $3^{\circ}$ cielo, allhora bisogneria andar con molta consideratione in esplicare le Scritture che paiono contrarie, e più tosto dire che non le intendiamo, che dire che sia falso quel che si dimostra" (Lettera a Paolo Antonio Foscarini, 12 aprile 1615, in [1] vol. XII, pp. 171-172).

Entrambi i contendenti, Galileo e Bellarmino, si rifacevano dunque al pensiero di Agostino! Peraltro, Bellarmino così prosegue: "Ma io non crederò che ci sia tal dimostratione, fin che non mi sia mostrata: né è l'istesso dimostrare che supposto ch'il sole stia al centro e la terra nel cielo, si salvino le apparenze, e dimostrare che in verità il sole stia nel centro e la terra nel cielo; perché la prima dimostratione credo ci possa essere, ma della seconda ho grandissimo dubbio, et in caso di dubbio non si dee lasciare la Scrittura Santa, esposta dai Santi Padri”. Tale posizione viene lodata come scientificamente corretta da Feyerabend [2], e certamente sarebbe piaciuta ad Agostino.

Per tornare al problema della forma del cielo, è chiaro che Agostino 
vede benissimo la contraddizione: "In realtà che c'è di tanto diverso e contrario quanto la superficie piana e distesa d'una pelle e la curva d'una volta convessa?" (De Gen. II, 9. 21). Tuttavia egli cerca comunque di cavarsi d'impaccio anche dal punto di vista letterale: "Se infatti una volta può chiamarsi correttamente non solo curva ma anche piatta, certamente anche una pelle può essere stesa non solo come una superficie piana ma anche in forma d'una tasca rotonda. In realtà sono una pelle non solo un otre ma anche una vescica" (9. 22). La spiegazione appare decisamente "stiracchiata", perché, anche se non poteva certo conoscere il theorema egregium che Gauss dimostrò nel 1827, Agostino aveva già ammesso che una volta e un piano sono ben diversi!

\section{IL TEMPO}

Uno dei temi più affascinanti della scienza e della filosofia è da sempre la natura del tempo. Diverse concezioni si sono affrontate nel pensiero dei popoli: dal tempo illimitato ab eterno al tempo circolare e ricorrente. Agostino sposa quella del tempo limitato. Oltre che nel De Genesi, vi sono bellissime pagine nelle Confessioni che trattano del tempo. Non si può non citare la famosa dichiarazione, che esprime la difficoltà di affrontare questo concetto: "Quid est ergo tempus? si nemo ex me quaerat, scio; si quaerenti explicare velim, nescio / Cos'è dunque il tempo? Se nessuno m'interroga, lo so; se volessi spiegarlo a chi m'interroga, non lo so" (Conf. XI, 14. 17).

Poco oltre (Conf. XI, 20. 26) Agostino descrive l'aspetto psicologico del tempo: "Tempora sunt tria: praesens de praeteritis, praesens de praesentibus, praesens de futuris. Sunt enim haec in anima tria quaedam et alibi ea non video. Praesens de praeteritis memoria; praesens de praesentibus contuitus; praesens de futuris expectatio / I tempi sono tre: presente del passato, presente del presente, presente del futuro. Questi tre tempi sono nella mia anima e non li vedo altrove. Il presente del passato è la memoria; il presente del presente, la percezione; il presente del futuro l'attesa".

Il rapporto tra tempo e movimento era già stato investigato da numerosi filosofi.

Platone (Timeo, 37d) parla del tempo come di "un'immagine mobile dell'eternità ... che procede secondo il numero, e che noi abbiamo chiamato tempo"; e spiega poi che l'era e il sarà sono forme di 
tempo, ma che all'eternità si addice solo l'è. Agostino ribadisce (Conf. XI, 11. 13) che "nell'eternità nulla passa, ma tutto è presente, a differenza del tempo, mai tutto presente".

\subsection{La creazione del tempo}

Ma inframmezzate con queste considerazioni psicologiche vi sono altri ragionamenti più scientifici.

Il tempo per Agostino è stato creato assieme al mondo. Prima non esisteva, anzi, non c'è un prima, perché l'inizio è stato appunto l'atto della creazione. "Ecco come rispondo a chi chiede: «Cosa faceva Dio prima di fare il cielo e la terra?». Non rispondo come quel tale, che, dicono, rispose, eludendo con una facezia l'insidiosità della domanda: «Preparava la geenna per chi scruta i misteri profondi». Altro è capire, altro è schernire. Io non risponderò così. Preferirei rispondere: «Non so ciò che non so», anziché in modo d'attirare il ridicolo su chi ha posto una domanda profonda, e la lode a chi diede una risposta falsa. Invece dico che tu, Dio nostro, sei il creatore di ogni cosa creata; e se col nome di cielo e terra s'intende ogni cosa creata, arditamente dico: «Dio, prima di fare il cielo e la terra, non faceva alcunché». Infatti, se faceva qualcosa, che altro faceva, se non una creatura?" (Conf. XI, 12. 14).

\subsection{Tempo e movimento}

Nel De Genesi (V 5. 12) Agostino afferma: "Così, dunque, il decorso del tempo iniziò con il movimento - mutamento delle creature; invano quindi si ricerca il tempo prima della creazione, come se fosse possibile trovare il tempo prima del tempo. Se infatti non ci fosse alcun movimento delle creature, spirituali o corporali, mediante il quale al passato succede il futuro attraverso il presente, non vi sarebbe affatto il tempo. La creatura poi non potrebbe muoversi - mutarsi, se non esistesse. Il tempo dunque è iniziato con la creazione anziché la creazione col tempo; l'uno e l'altra poi provengono da Dio, poiché da lui, grazie a lui e in lui sono tutte le cose".

Aristotele afferma che "il tempo è misura di un movimento secondo il prima e il poi" (Phys. IV, 10-11). Al contrario, Plotino dichiara che "quello che viene misurato dalla rotazione celeste, ossia quello che viene rivelato, sarà propriamente il tempo; ma questo non è creato da 
quella rotazione, ma semplicemente è messo in luce. Per tale motivo [alcuni filosofi], anziché sostenere che il movimento è la misura del tempo e aggiungere quale cosa sia effettivamente misurata dal movimento, tacendo il fatto che ciò avviene solo per accidente e per un certo tipo di tempo, furono portati a credere che il tempo fosse la misura del movimento e a invertire l'ordine dei fatti" (Enn. III, 7, 12-13).

\subsection{Movimento degli astri}

Se il tempo è legato al movimento, il più significativo movimento che lo esprime è quello degli astri, che danno anche lo spunto per le prime unità di misura del tempo stesso: anno, mese e giorno, che Agostino, nel De Genesi (II, 14-15) descrive con puntigliosa esattezza, spiegando gli anni bisestili, le fasi della luna e la sua forma sferica, che evidentemente qualcuno, ancora ai suoi tempi, credeva fosse piatta o di forma mutevole.

Si noti che il giorno della Creazione non può che essere allegorico, perché "quando da noi è notte, la presenza della luce illumina le altre parti del mondo che il sole percorre prima di tornare dalla parte ove tramonta a quella ove sorge; per questo motivo nello spazio di tutte le ventiquattro ore c'è sempre, lungo il percorso circolare del sole, una parte [della terra] ov'è giorno e un'altra ov'è notte" (De Gen. I, 10, 21); e certo Dio non stava in un particolare punto della Terra quando creò il mondo!

Tuttavia per Agostino (Conf. XI, 23, 29-30) il tempo non è tale movimento: "Ho udito dire da una persona istruita che il tempo è, di per sé, il moto del sole, della luna e degli astri; e non assentii. Perché il tempo non sarebbe piuttosto il moto di tutti i corpi? Qualora si arrestassero gli astri del cielo, e si muovesse la ruota del vasaio, non esisterebbe più il tempo per misurarne i giri e poter dire che hanno durate uguali, oppure, se si svolgono ora più lenti, ora più veloci, che gli uni sono più lunghi, gli altri meno?".

E prosegue, di nuovo con un esempio tratto dalla Bibbia, cui ho accennato prima citando il card. Bellarmino: "Dunque non mi si dica che il tempo è il movimento dei corpi celesti. Quando il sole si fermò all'appello di un uomo per dargli modo di concludere una battaglia vittoriosa, il sole era fermo, ma il tempo procedeva, tant'è vero che la battaglia fu condotta e finita nello spazio di tempo ad essa sufficiente”.

Quest'ultima osservazione naturalmente si riferisce al famoso epi- 
sodio narrato nel libro di Giosuè 10, 12-14: "Quel giorno, quando il Signore diede a Israele la vittoria sugli Amorrei, Giosuè pregò il Signore e gridò alla presenza di tutti gli Israeliti: 'Sole, fermati su Gabaon! e tu, luna, sulla valle di Aialon! Il sole si fermò, la luna restò immobile, un popolo si vendicò dei suoi nemici'. Questo avvenimento è descritto nel 'Libro del Giusto'; per quasi un giorno intero il sole restò in alto in cielo, senza avviarsi al tramonto. Un giorno come quello non c'è mai stato né prima né dopo di allora, quando il Signore ubbidì a un essere umano e combatté al fianco d'Israele".

È di nuovo interessante notare come lo stesso esempio sia preso da Galileo, nella Lettera a don Benedetto Castelli, a sostegno della teoria eliocentrica. Molto più semplice, egli argomenta con un lungo ma convincente ragionamento, è pensare che sia stata la Terra ad aver interrotto la sua rotazione e non il Sole, e con esso tutta la volta celeste e il primo mobile, responsabile del movimento diurno degli astri.

\section{L'ASTRONOMIA E L'ASTROLOGIA}

Agostino, così esperto di astronomia, rifiutava con assoluta determinazione l'astrologia. Da giovane invero aveva consultato gli astrologi, come ammette nelle Confessioni (IV, 3): "non rinunziavo ancora a consultare quegli imbroglioni che sono detti mathematicos", ma più tardi si era ricreduto e aveva "rifiutato le false divinazioni e le empie fantasticherie degli astrologi (mathematicorum)" (Conf., VII, 6).

Questo rifiuto era dovuto, naturalmente, alle Scritture, che in vari passi condannano tali prassi, ma era già implicito nella discussione sui gemelli.

Secondo Agostino "il concepimento dei gemelli, che si attua con una sola unione, come attestano i medici, la cui scienza è molto più sicura ed evidente, avviene in un tempo così rapido da non oltrepassare due secondi". E siccome gli astrologi dei secondi non si curano ("Minutas autem minutarum iam in constellationibus ... non inveniunt", 83 questioni diverse, n. 45 - Contro gli astrologi), due gemelli dovrebbero avere lo stesso destino, il che non è. Sono argomentazioni che avrebbe tranquillamente potuto riprendere da Cicerone (De Divinatione, II, 87-99), ma volendosi basare sull'evidenza fattuale diretta riporta un fatto di cronaca, raccontato da un certo Firmino (Conf. VII, 6, 8-10), per cui due ragazzi, nati nello stesso momento, avevano 
avuto vite differenti. Del resto, annota, anche Esaù e Giacobbe erano gemelli!

Concludendo queste argomentazioni, Agostino osserva acutamente: "Si dice che [gli astrologi] hanno predetto molte cose vere, ma questo dipende dal fatto che gli uomini dimenticano le loro falsità ed errori. Unicamente preoccupati di quanto si accordava alle loro aspettative, dimenticano ciò che non corrispondeva e ricordano solo gli avvenimenti che capitano accidentalmente, non per arte divinatoria, del tutto inesistente, ma per qualche fortuita coincidenza”. Si tratta di un fenomeno psicologico che possiamo verificare ancora ai giorni nostri, e non solo riguardo all'astrologia (che incredibilmente sopravvive ancora) ma anche a riguardo di molti altri discorsi antiscientifici che si diffondono con la complicità dei mezzi di comunicazione e che nessuno è in grado di arginare.

\section{LE SCIENZE NATURALI E LA FISICA}

Vediamo ora alcuni esempi di fenomeni fisici descritti da Agostino. Anche se sovente le descrizioni sono corredate da spiegazioni arzigogolate e assolutamente prive di valore scientifico, esse dimostrano uno spirito di osservazione degno di nota e una vasta conoscenza delle cognizioni dell'epoca. Vorrei anche notare che Agostino, da maestro di retorica qual era, puntigliosamente esamina tutte le spiegazioni alternative e tutti i significati che si possono dare alle parole, perché ovviamente il contesto apologetico non consente la precisione tipica del linguaggio scientifico.

\subsection{Le acque sopra il cielo e il ciclo delle acque}

Un primo esempio si trova nel commento al secondo giorno della creazione: "Dio inoltre disse: Vi sia un firmamento in mezzo alle acque e tenga separate le acque dalle acque. E così avvenne. E Dio fece il firmamento e separò le acque ch'erano al di sopra del firmamento. E Dio chiamò 'cielo' il firmamento. E Dio vide ch'essa è cosa buona. E venne sera e poi mattina: secondo giorno".

Agostino (De Gen. II, 1.2), dopo aver notato che "per loro natura, queste acque non possono trovarsi al di sopra del cielo stellato poiché, a causa del loro peso, sono costrette a scorrere sulla terra oppure, 
evaporando, ad esser trasportate nell'atmosfera vicina alla terra" espone alcune esperienze di fisica. Dapprima nota (2.5) che "un recipiente immerso [nell'acqua] con la bocca rivolta verso il basso non può riempirsi d'acqua: questa è una prova sufficiente che l'aria tende per sua natura verso l'alto. Il recipiente sembra bensì vuoto ma si dimostra esser pieno d'aria quando lo s'immerge nell'acqua con la bocca rivolta verso il basso; in realtà poiché [l'aria] non trova una via d'uscita attraverso la parte rivolta verso l'alto e, data la sua natura, non può passare sotto l'acqua penetrandovi a forza, la respinge dal recipiente ch'è pieno e non ve la lascia entrare". Poi descrive il gorgoglio dell'aria quando fuoriesce perché l'acqua entra nel vaso inclinato.

Peraltro si lascia anche andare a considerazioni del tutto prive di fondamento quando per esempio attribuisce alle acque che stanno sopra il cielo la capacità di raffreddare Saturno, che, secondo lui, siccome nel suo moto diurno si muove più rapidamente degli altri dovrebbe piuttosto essere caldo. (De Gen. II, 5, 9).

Nel libro incompiuto del De Genesi, in cui si riprendono molti dei ragionamenti degli altri libri, è descritto con precisione il ciclo delle acque (14. 47): "D'altra parte la Scrittura, parlando delle sorgenti e dei fiumi, non dice neppure come sono stati creati. Ora, coloro che indagano e discutono questi fenomeni, affermano che, per via del movimento superficiale dell'aria, dal mare si solleva invisibilmente il vapore d'acqua dolce evidentemente per via di queste esalazioni che noi non possiamo osservare in alcun modo: queste esalazioni si condensano in nubi, e in tal modo la terra bagnata dalle piogge emette a goccia a goccia l'acqua in caverne assai occulte e ve la trasuda nella misura in cui, dopo essersi raccolta e penetrata per diversi meati, scaturisce in sorgenti sia piccole sia capaci di formare dei fiumi.

Gli scienziati sostengono che di questo fenomeno è prova il fatto che l'evaporazione dell'acqua marina fatta bollire e raccolta in una serpentina presenta, a chi l'assaggia, acqua dolce. A tutti inoltre appare evidente che le sorgenti diminuiscono di portata poiché risentono l'effetto della scarsezza delle piogge".

\subsection{Il cervello}

Nel settimo libro del De Genesi Agostino tratta della creazione dell'anima, e del suo rapporto col corpo. In particolare descrive la 
struttura del cervello, che secondo la medicina dell'epoca era costituito da tre ventricoli: "il primo vicino al volto, dal quale si dipartono tutti i nervi sensori; il secondo è quello posteriore situato presso la base del cervello, che regola tutti i movimenti; il terzo è sito tra gli altri due, ove gli scrittori dimostrano che ha sede la memoria" (De Gen. VII, 18. 24).

E prosegue con una dimostrazione molto moderna di tale partizione: "L'esistenza di siffatti ventricoli, al dire di quegli scrittori, è dimostrata da segni sicuri, in casi in cui quelle rispettive zone del cervello sono state affette da una malattia o da un difetto patologico. Anche quando sono menomate le funzioni della sensazione o il movimento delle membra o il ricordo dei movimenti del corpo, [i medici] indicano assai chiaramente la funzione di ciascuno dei ventricoli e, applicando a questi la cura [opportuna], hanno appurato a quale delle zone cerebrali ha giovato la cura apprestata". La medicina moderna ovviamente ha strumenti molto fini per isolare le zone attive del cervello in ciascuna delle operazioni che compie, ma concettualmente il metodo è corretto e funzionale.

\subsection{Qualche altro errore}

Alcune delle teorie scientifiche esposte da Agostino, oltre al geocentrismo sono (per noi moderni!) palesemente erronee.

Per esempio, egli credeva alla generazione spontanea di animali, seguendo sia la Bibbia che Aristotele; ma per confutare tale teoria si dovette attendere il XVII secolo, con le Esperienze intorno alla generazione degl'insetti di Francesco Redi del 1668 seguito, nel secolo successivo, dal Saggio di osservazioni Microscopiche sul Sistema della Generazione de' Signori di Needham e Buffon di Lazzaro Spallanzani (1765) e infine, ancora cent'anni dopo, da Louis Pasteur, che nel 1864 chiuse la questione con una dimostrazione premiata dall'Accademia delle Scienze di Parigi.

Anche nella teoria della visione era in errore, pensando che il raggio si propagasse dagli occhi alla sorgente luminosa e non viceversa. Questo errore forse nasce da una interpretazione fisica del linguaggio puramente geometrico dell'ottica di Euclide, che ovviamente si mette dal punto di vista dell'osservatore per descrivere l'angolo o il cono sotto cui si vede l'oggetto; ma Euclide non aveva alcuna intenzione di fare una teoria fisica della visione. 


\section{LA MATEMATICA}

Non intendo spendere troppe parole sulla matematica di Sant'Agostino, avendone già trattato diffusamente in una relazione svolta nel convegno Matematica e Letteratura 2 (Fisciano, SA, 6-8 aprile 2016) e riportata negli atti di quel convegno [3].

Non posso però non ricordare che non solo Agostino aveva una conoscenza non banale della aritmetica dei suoi tempi (numeri primi, perfetti, triangolari ecc.), ma che al solito ne faceva strumento di interpretazione allegorica della Scrittura.

Per esempio, dopo aver dimostrato che 6 è un numero perfetto, e avendo anche chiosato, senza uno scopo specifico, ma per il puro piacere di esprimere delle proprietà matematiche, che pure 28 lo è e che i numeri perfetti si diradano sempre più (di fatto, all'epoca se ne conoscevano solo quattro: 6, 28, 496 e 8128), egli spiega così i tempi della creazione: "Noi quindi non possiamo dire che il sei è un numero perfetto per il fatto che Dio ha compiuto tutte le sue opere in sei giorni, ma possiamo dire che Dio ha compiuto le sue opere in sei giorni per il fatto che il sei è un numero perfetto. Questo numero perciò sarebbe perfetto anche se queste opere non ci fossero state; se invece esso non fosse perfetto, Dio non avrebbe compiuto le sue opere attenendosi a questo numero" (De Gen. IV, 7.14). Naturalmente, i sei giorni sono puramente allegorici: "Ecco perché, quando pensiamo alla creazione primordiale degli esseri, cioè alle opere dalle quali Dio si riposò il settimo giorno, non dobbiamo immaginare quei giorni come i nostri giorni solari né l'operazione di Dio come se fosse l'attività con cui ora compirebbe qualcosa nel tempo, ma dobbiamo pensare piuttosto il modo con cui operò ciò da cui cominciò il tempo, il modo cioè con cui fece tutte le cose simultaneamente dando loro anche un ordine risultante non da intervalli temporali ma dalla connessione delle cause; in tal modo gli esseri creati simultaneamente furono anche portati a compimento alla perfezione mediante la ripetizione del "giorno" [della creazione] fatto presente per sei volte" (De Gen., V, 5. 12).

Così i 153 grossi pesci della pesca miracolosa sono per lui simbolo della Chiesa dei santi: infatti "se al numero dieci, proprio della legge, aggiungiamo il numero sette, proprio dello Spirito Santo, abbiamo diciassette. Se si scompone questo numero in tutti i numeri che lo formano, e si sommano tutti questi numeri, si ha come risultato centocin- 
quantatré”. In termini matematici, il numero triangolare di 17 è appunto $1+2+3+\ldots+17=153$ (in Io. Ev. 122,8 ).

Ma il punto in cui Agostino si distacca dalla tradizione matematica antica e in qualche modo precorre la modernità è nella concezione della totalità dei numeri non più solo come potenziale, ma come una infinità attuale, compresente nella mente di Dio, e quindi per ciò stesso concepibile anche dall'uomo.

"Riguardo poi all'altra loro teoria che neanche con la scienza di Dio può essere rappresentato l'infinito, rimane loro che osino affermare, immergendosi nell'abisso profondo della irreligiosità, che Dio non conosce tutti i numeri.

È assolutamente certo che il numero è infinito, perché qualunque sia il numero che si prende come limite, non dico che è possibile aumentarlo di un'unità, ma per quanto sia grande e comprensivo di una indefinita quantità numerica, in base all'idea stessa del numero, non solo si può raddoppiare, ma anche moltiplicare per se stesso. Infatti qualsiasi numero è così determinato dalle sue proprietà che non v'è numero eguale ad un altro. Sono dunque disuguali per quantità e qualità, ognuno è finito, e tutti insieme sono infiniti.

Dio dunque non conoscerebbe a causa dell'infinità la totalità dei numeri e la sua scienza arriverebbe fino a una certa quantità numerica e ignorerebbe il resto? Non lo potrebbe dire neanche il più insensato".

Molti altri esempi potrebbero essere riportati a testimonianza della accuratezza della descrizione dei fenomeni fisici e matematici da parte di Sant'Agostino, oltre che della eleganza del suo eloquio, ma spero che questi bastino a illustrare questo aspetto della sua personalità di uomo capace di coniugare così felicemente la fede e la ragione.

\section{NOTA BIBLIOGRAFICA}

I testi di Agostino sono tratti, per comodità, dal sito ufficiale [4], che riporta l'intero corpus agostiniano sia in latino che in italiano, oltre che in altre lingue moderne. Per questo non vengono riportati i testi cartacei consultati, anche se ricchi di pregevole materiale commentario.

1. Galilei G., Opere, a cura di A. Favaro, Giunti-Barbera, Firenze 1968.

2. Feyerabend P., Galileo e la tirannia della verità, L'Astronomia n. 71, 1987, 28-36.

3. Citrini C., Sant'Agostino e la matematica, in Parole, formule, emozioni, a cura di Maroscia P., Toffalori C., Tortoriello F.S. Vincenzi G., 145-181, UTET, 2018.

4. www.augustinus.it 
